\title{
Comparison of Betamethasone 17-Valerate Ointment with Fluocortolone and Fluocortolone Caproate Ointment
}

\author{
DOWLING D. MUNRO,* M.B., M.R.C.P.; H. T. H. WILSON,† M.D., F.R.C.P. \\ ELLEN L. RHODES, $\ddagger$ M.R.C.P., D.C.H.
}

Brit. med. F., 1967, 4, 275-276

Topical corticosteroids have revolutionized the therapy of many dermatoses. The value to the dermatologist of commercially available preparations reflects many factors, including the cost, potency, therapeutic efficiency, and cosmetic acceptability of the medicaments. Furthermore, since the pharmacology of most corticosteroids is similar, and the newer fluorinated compounds are of high potency, it becomes increasingly difficult for the clinician to assess the value of different preparations on the market.

Clinical impressions resulting from uncontrolled observations can be misleading, but properly conducted double-blind trials avoid the errors arising from bias on the part of the physician or patient. The present investigation compares the value of betamethasone 17-valerate ointment (Betnovate), with fluocortolone and fluocortolone caproate ointment (Ultralanum Plain). It is not intended as a comparison of the value of the constituent steroids or their bases, but as a clinical comparison of the two preparations as marketed in the United Kingdom.

\section{Methods and Materials}

Double-blind trial packs were prepared in pairs marked left and right. Betnovate ointment-that is, $0.1 \%$ betamethasone 17-valerate-and Ultralanum Plain ointment-that is, a mixture of $0.25 \%$ fluocortolone and $0.25 \%$ fluocortolone caproatewere randomly allocated to the left and right sides: each tube contained $30 \mathrm{~g}$. of the relevant ointment. Each paired pack was numbered and the code given to the clinicians in sealed envelopes, which remained closed throughout the trial.

Three dermatologists from different centres co-operated in the trial. The same dermatologist saw the patient before and after treatment, and recorded details of the cases on a standard form.

Individuals admitted to the trial were suffering from either eczema or psoriasis and were treated as outpatients. Only those having approximately symmetrical involvement of right and left sides were studied, and there was random selection in so far as all suitable patients referred to the clinics were consecutively included in the trial. Patients were instructed to apply the test ointments twice daily; some, where it was felt to be necessary, were told to occlude affected areas with polyethylene film. Mild sedatives or antihistamines were administered if indicated.

Detailed records of individual patients included name, age, sex, diagnosis, and sites and extent of the disease, together with the history and duration of previous therapy. In the trial the dermatologist's assessment ("objective") and the patient's views ("subjective") of the relative improvement between sides were compared and separately recorded as "healed," "improved,"

- Research Fellow, St. Mary's Hospital, London W.2; Consultant Dermatologist, Mount Vernon Hospital, Northwood, Middlesex, and Edgware General Hospital, Edgware, Middlesex.

t Consultant Dermatologist, Central Middlesex Hospital, London N.W.10, and Royal Northern Hospital, London N.7.

₹ Consultant Dermatologist, St. Helier and Kingston Group Hospitals, Surrey. "static," or " worse." The dermatologist assessed improvement by observing reduction in erythema, scaling, induration, and oedema of the diseased skin. The patient assessed the changes in itching or other symptoms. The two sides were simply compared, no attempt at quantitative assessment being made. The patients were normally reviewed after seven days, though some were seen more frequently, and a few at slightly longer intervals.

When the investigation on each patient was concluded, the completed form was returned to a co-ordinating secretary, who decoded the results and plotted them consecutively on previously constructed restricted sequential analysis graphs (Armitage, 1960) having a design designated by the factors

$$
2 \alpha=0.05,1-\beta=0.95, \theta_{1}=0.85 \text {. }
$$

A total of 109 patients were included in the trial. Of these, 66 had eczema and 43 had psoriasis. Their ages ranged from 6 months to 76 years and the sex ratio was 48 males to 61 females. These two groups were subdivided into those occluded with polyethylene, and those treated without polyethylene. Two patients who were initially included in the trial failed to carry out the instructions given by the clinicians and were therefore excluded from the investigation.

The total numbers treated are shown in the Table. Statistical investigations, by means of restricted sequential analysis, of the results in the first two groups are shown in Fig. 1 (eczema) and Fig. 2 (psoriasis). This method of statistical evaluation excludes patients in whom both sides responded equally (" tied pairs"), but these are shown in the Table.

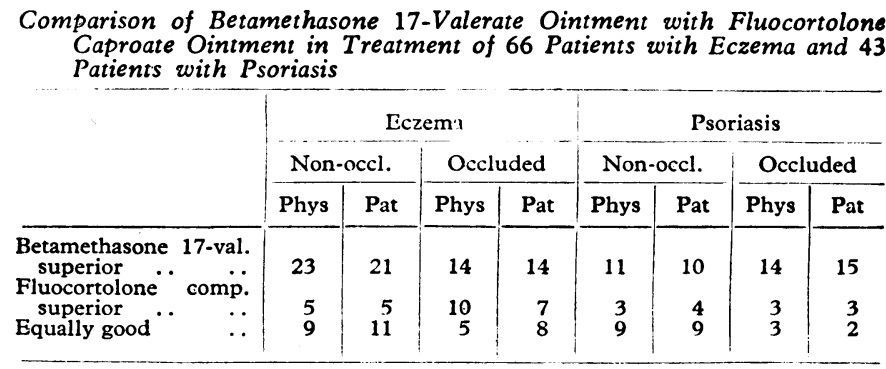

Phys $=$ Physician's opinion. Pat $=$ Patient's opinion.

In the eczema group (Table and Fig. 1) betamethasone $17-$ valerate was shown to be statistically superior at the $5 \%$ level in patients treated without occlusion, and the boundary was reached after 30 patients. In patients treated with plastic occlusive dressings the "no significant difference" boundary was reached after 25 patients had been investigated.

In the psoriasis group (Table and Fig. 2) patients treated either with or without polyethylene occlusion responded better to betamethasone 17-valerate ointment than to the fluocortolone compound ointment. The statistically significant boundary at the $5 \%$ level was reached after 20 cases were treated with occlusion and 17 cases without occlusion.

The patient's own opinions, also included in the Table, show a remarkable correlation between them and their physicians in 
the assessment of response. On the whole, when they differed in their views on which side was most improved, the variation between sides was slight.

\section{Discussion}

Under the conditions of the trial betamethasone 17-valerate ointment was impressively better than the fluocortolone preparation in the treatment of psoriasis, whether occluded or not, and in eczema when used without occlusion. In other independent trials-mainly psoriasis treated with occlusion-fluocortolone alone, and also with fluocortolone caproate and clemizolehexachlorophane, has similarly been shown to be less effective than betamethasone 17-valerate (Sneddon, 1966, 1967). The use of occlusive dressings increases humidity and the temperature of the skin, resulting in increased percutaneous absorption. This tends to mask differences in efficiency of steroid

were not too subtle for the patients to be conscious of them A multicentred trial such as the present one has the advantage that minor centre-by-centre differences in the objective assessments tend to be corrected. In one centre, investigation of the eczema non-occluded group of cases was continued after the main trial was completed, and showed a similar pattern of significance for betamethasone 17-valerate ointment after 20 cases had been studied.

There are difficulties in assessing statistically the biological response to two drugs, and sequential analysis has disadvantages if the number of "tied pairs" of equal response is large. Variation in the pattern of the graph can occur, depending on the order in which results are plotted. In this investigation the results were plotted strictly according to the date of reaching a conclusion for a particular patient in the trial. If two cases were completed on the same date, then the lower number of the paired packs was plotted first. The advantages of sequential analysis are that, by prior determination of the limits of the trial, a statistically acceptable assessment is achieved with the minimum number of patients, and that such data can be easily presented.

It should be emphasized that this trial does not compare the active constituents of the two ointments but was specifically designed to compare two commercially available products. The total strengths of the steroid constituents differed as did the bases, the betamethasone 17-valerate preparation being in a soft paraffin and hydrogenated lanolin base, while the fluocortolone preparation was in a soft paraffin aqueous emulsion containing 30\% water. One preparation (betamethasone 17-valerate ointment), was superior to the other (fluocortolone RESTRICTED SEOUENTIAL ANALYSIS $\theta_{1}=0.85,2 \alpha=0.05,1-\beta=0.95$

\begin{tabular}{l}
-.... TREATED WITHOUT OCCLUS \\
\hline
\end{tabular}

FIG. 1.-Statistical investigation of patients with eczema. FIG. 2.-Statistical investigation of patients with psoriasis.

preparations of different potency (Thomsen, 1966), and was demonstrated in the present trial by the group of patients suffering from eczema and treated with occlusion, in whom the two preparations gave a similar response. Topical corticosteroids when applied in large amounts under occlusive dressings may depress the hypothalamic-pituitary-adrenal axis (Scoggins, 1962 ; Strange and Hjorth, 1965 ; Munro et al., 1967). In small quantities, such as were used in this investigation, systemic effects would be minimal.

Psoriasis is more resistant than eczema to treatment with corticosteroids, and even hydrocortisone, which gives little benefit in psoriasis, usually produces considerable improvement in eczema. It would appear, therefore, that response in psoriasis is a more stringent test of the efficacy of a corticosteroid than response in eczema. The superiority of betamethasone 17valerate ointment in this context is noteworthy. The mechanism of action of corticosteroids on psoriasis is unknown but probably relates to the prolongation of epidermal cell turnover times which has been shown to occur in normal skin treated with a corticosteroid (Munro, 1967). However, from a clinical viewpoint corticosteroids, while useful in some psoriatic cases, are not the treatment of choice in many cases owing to their tendency to relapse when therapy is discontinued-presumably as the result of epidermal cell proliferation returning to the psoriatic pattern.

Differences in the response to the two preparations were slight in many patients, and it had been noted previously that experienced observers tended to record differences which were not obvious to clinicians less accustomed to observing dermatological disease. However, the correlation between objective and subjective findings was good, thus emphasizing that differences caproate ointment) in three out of the four groups studied, but this does not mean there is no place in the market for the compound that appeared in this short-term trial to be less effective. Individuals respond differently, and some steroid compounds seem to become less effective as treatment continues. The dermatologist is often looking for different potent compounds to use while the natural tendency for the skin to heal is occurring Both the ointments tested contain powerful corticosteroids and, as such, have their use.

\section{Summary}

In a double-blind trial $0.1 \%$ betamethasone 17 -valerate ointment (Betnovate) has been compared with $0.25 \%$ fluocortolone and $0.25 \%$ fluocortolone caproate compound ointment (Ultralanum Plain) in the treatment of eczema and psoriasis, with and without polyethylene occlusion. The betamethasone 17-valerate ointment was significantly better than the fluocortolone ointment in patients with eczema treated without occlusion, and in psoriasis, with or without occlusion. No significant difference was demonstrated in cases of eczema treated with the ointments and occluded with polyethylene.

\section{REFERENCES}

Armitage, P. (1960). Sequential Medical Trials. Oxford.

Munro, D. D. (1967). Awaiting publication

Munro, D. D. (1967). Awel, M., and James, V H. T. (1967). Proc. 13th int. Congr. Derm. In press.

Scoggins, R. B. (1962). F. invest. Derm., 39, 473

Sneddon, I. B. (1966). Brit. 7. Derm., 78, 554

(1967). Ibid., 79, 122.

Strange, H. A., and Hiorth, N. (1965). Proc. 17th Meeting Fenno-Scand. Ass. Derm Copenhagen

Thomsen, K. (1966). Ugeskr. Lag., 128, 1391. 